Pacific Journal of Mathematics

ASYMPTOTIC PERTURBATION SERIES FOR 


\title{
ASYMPTOTIC PERTURBATION SERIES FOR CHARACTERISTIC VALUE PROBLEMS
}

\author{
C. A. Swanson
}

1. Introduction. Ordinary linear differential operators of the type

$$
L=p_{n} \frac{d^{n}}{d x^{n}}+p_{n-1} \frac{d^{n-1}}{d x^{n-1}}+\cdots+p_{0} \quad(n \geq 2)
$$

will be under consideration on a half-open real interval $(0, b](b>0)$, designated as the basic interval. The coefficients $p_{j}=p_{j}(x)(j=0,1, \cdots, n)$ are real-valued, continuous functions possessing $j$ continuous derivatives on $(0, b]$, and $p_{n}(x) \neq 0$ on $(0, b]$. The point $x=0$ is supposed to be a singularity for $L$.

The basic operator over the Hilbert space $\mathscr{L}^{2}(0, b)$ will be obtained as a restriction of $L$ to a domain consisting of functions which are sufficiently differentiable and which satisfy certain boundary conditions. When $L$ coincides with its Lagrangian adjoint, conditions are known [1] under which an operator like this is self-adjoint over $\mathscr{L}^{2}(0, b)$. Our attention will not be focused on a self-adjoint operator, however, but on a basic operator which has at least one isolated point in its spectrum.

The investigation here concerns the spectrum of a perturbed operator. Let $[\varepsilon, b]$ denote a closed subinterval of the basic interval, where $\varepsilon$ is a small positive number. A perturbed operator $A_{\varepsilon}$ is a restriction of $L$ to a domain in $\mathscr{L}^{2}(\varepsilon, b)$ consisting of functions which are suitably differentiable on $[\varepsilon, b]$, and which satisfy homogeneous boundary conditions at the endpoints $x=\varepsilon$ and $x=b$. Then a set of perturbed operators is obtained when $\varepsilon$ varies. It will be shown that for each characteristic value $A$ of the basic operator, there is a characteristic value $\lambda(\varepsilon)$ of the perturbed operator $A_{\varepsilon}$ which converges to $\Lambda$ as $\varepsilon \rightarrow 0$; and furthermore that $\lambda(\varepsilon)$ can be represented by an asymptotic expansion, valid as $\varepsilon \rightarrow 0$.

An asymptotic expansion for the characteristic function $u$ corresponding to $\lambda(\varepsilon)$ will also be established. In particular, the asymptotic form $u(x)=U(x)[1+o(1)]$ will be obtained, in terms of the characteristic function $U$ of the basic operator corresponding to 1 , valid uniformly for $x$ contained in a certain closed subset of $[\varepsilon, b]$ as $\varepsilon \rightarrow 0$. Evidently such an asymptotic form cannot hold uniformly near the zeros of $U$, nor can it hold near the boundary $x=\varepsilon$ since $u$ is forced to satisfy a boundary condition at $x=\varepsilon$. The procedure used herein permits a representation for the characteristic functions to be obtained in the "boundary layer"

Received November 5, 1958. 
near $x=\varepsilon$, as well as the uniform result stated above.

The distinctive feature of the problem under consideration is that the domain of the basic operator has been perturbed to a "slightly different" domain, depending upon the small parameter $\varepsilon$. In the usual perturbation theories, the operator $L$ itself is perturbed: the perturbed operator is defined formally by the relation $A_{\varepsilon}=T_{0}+\varepsilon T_{1}+\varepsilon^{2} T_{2}+\cdots$. One then develops the characteristic values and characteristic functions of $A_{\varepsilon}$ in convergent or asymptotic power series in $\varepsilon$ as $\varepsilon \rightarrow 0$ [5]. In the present case, when the perturbation arises from the domain of the operator, power series expansions are not valid in general, and instead more general types of expansions will be obtained.

The present method consists of comparing the solutions of the perturbed problem with those of the basic problem by means of an integral equation of Volterra's type. The kernel of the integral equation has a well-known [2, 4] representation as the quotient of determinants of order $n$. A specific representation for solutions of the perturbed problem can then be obtained.

The analogous problem for second order self-adjoint operators has been treated previously [9]. The present results will correspond to the class 1 problems in [9], for which there exist linearly independent solutions of (1.1) which can be ordered according to their asymptotic behaviour near $x=0$. Asymptotic expansions for perturbed characteristic values and functions are then obtained, and the main theorems are enunciated in $\$ 4$.

2. Definitions. The asymptotic terminology to be used in the sequel will first be described. This follows Van der Corput [10]. Asymptotic expansions of functions $f(\varepsilon)$ for small values of the positive, real variable $\varepsilon$ will be under consideration. Let $\delta(\varepsilon)$ be a positive function of $\varepsilon$ with the property that $\delta=o(1)$ as $\varepsilon \rightarrow 0$. The function $\delta$ will be called a scale.

DEFINITION 1. The formal series $\sum f_{i}$ is said to be an asymptotic expansion for the function $f(\varepsilon)$ with scale $\delta$, as $\varepsilon \rightarrow 0$, if the order relation $f-f_{1}-f_{2}-\cdots-f_{i}=O\left(\delta^{i}\right)$ holds for each $i=1,2, \cdots$.

The notation $f \sim \sum f_{i}$ signifies that $\sum f_{i}$ is an asymptotic expansion for $f$.

Suppose that $f$ and $f_{i}$ are functions not only of $\varepsilon$, but of an additional real variable $x$ on an interval $I$, which may depend on $\varepsilon$. Let $\delta$ be a function of $\varepsilon$ and $x$ with the property that $\delta=o(1)$ as $\varepsilon \rightarrow 0$, uniformly for $x \in I$. Then a uniform asymptotic expansion for $f$ is defined as follows. 
DEFINITION 2. The series $\sum f_{i}$ is said to be an asymptotic expansion for $f$ with scale $\delta$ as $\varepsilon \rightarrow 0$, valid uniformly for $x \in I$, if for some function $\alpha(x, \varepsilon)$ the order relation $f-f_{1}-f_{2}-\cdots-f_{i}=O\left(\alpha \delta^{i}\right)$ holds uniformly for $x \in I$ for each $i=1,2, \cdots$.

The differential operator (1.1) is under consideration on the real interval $(0, b](b>0)$. It will be assumed without essential loss of generality that

$$
p_{n}(x)=-1, \quad p_{n-1}(x)=0 \quad 0 \leq x \leq b .
$$

All points in the interval $(0, b]$ are supposed to be ordinary points of $L$ and $x=0$ is supposed to be a singular point.

Let $\mathfrak{S}$ denote the Hilbert space $\mathscr{L}^{2}(0, b)$, and let $($, ) and $\|||$ denote the inner product and norm respectively in $\mathfrak{Y}$. Let $\mathcal{C}^{n-1}(a, b)$ denote as usual the class of real valued functions on $(a, b)$ possessing $n-1$ continuous derivatives. Certain transformations on $\mathfrak{S}$ will now be defined by suitably restricting the formal operator $L$.

Definition 3. The basic domain $\mathfrak{D}$ is the set of all $u \in \mathfrak{W}$ satisfying the following conditions.

(a) $u \in \mathscr{C}^{n-1}(0, b)$ and $u^{(n-1)}$ is absolutely continuous on every closed subinterval of $(0, b]$.

(b) $L u \in \mathfrak{S}$

(c) $u$ satisfies a set of $n-m(1 \leq m<n)$ linearly independent homogeneous boundary conditions at $x=b$, of the form

$$
\mathscr{\mathscr { S }}_{b k}[y]=\sum_{j=1}^{n} \beta_{k j} y^{(j-1)}(b)=0 \quad(k=1,2, \cdots n-m)
$$

for real numbers $\beta_{k j}$ [2].

The basic operator $T$ is then defined to have domain $\mathcal{D}$ and

$$
T u=L u \quad u \in \mathfrak{D} \text {. }
$$

It is possible that $T$ is already a self-adjoint transformation on the space $\mathfrak{S}$. When $n=2$, this corresponds to the limit point case in Weyl's classification of singular points [2, 11]. If $T$ is not self-adjoint, one can try to obtain all possible self-adjoint transformations by adjoining suitable conditions at $x=0$; but we are not going to be interested in forming self-adjoint transformations, and instead make some direct assumptions.

A set of $n$ linearly independent functions $V_{i}(x)$ on $(0, b)$ are said to be asymptotically ordered as $x \rightarrow 0$ when there exists a number $x_{0}>0$ so that each $V_{i}>0$ whenever $0<x \leq x_{0}$, and

$$
\lim _{x \rightarrow 0} \frac{V_{i}(x)}{V_{i+1}(x)}=0 \quad(i=1,2, \cdots n-1) .
$$


This property induces an ordering on the set $\left\{V_{i}\right\}$, which can be indicated by the chain

$$
V_{1} \prec V_{2} \prec \cdots \prec V_{n} .
$$

Let $\Lambda$ be a real number, and consider a fundamental set of solutions $U_{i}=U_{i}(x, \Lambda)(i=1,2, \cdots, n)$ of the differential equation $L y=\Lambda y$. Let

$$
\begin{gathered}
W_{i}=W_{i}(x, \Lambda)=(-1)^{n+i} \operatorname{det}\left(U_{h}{ }^{(k-1)}\right) \\
(h, i=1,2, \cdots, n ; h \neq i ; k=1,2, \cdots, n-1) .
\end{gathered}
$$

The following assumptions will be made.

Assumptions For at least one real number $\Lambda$, there is a fundamental set of solutions $U_{i}$ of the differential equation $L y=A y$ with the properties

(i) The set $\left\{U_{i}\right\}$ is asymptotically ordered as $x \rightarrow 0$.

(ii) For $j=i ; k, i=1,2, \cdots, n-1$; and $j=k=n, i=1,2, \cdots, n-1$,

$$
\frac{U_{i}(x)}{U_{n}(x)} \int_{x}^{b} W_{j}(t) U_{k}(t) d t=o(1) \text { as } x \rightarrow 0 .
$$

(iii) For some positive number $x_{0}$,

$$
\begin{gathered}
\left|U_{i}(x) W_{i}(t)\right| \leq\left|U_{n}(x) W_{n}(t)\right| \\
\left(0<x \leq t \leq x_{0}\right) \quad(i=1,2, \cdots, n-1)
\end{gathered}
$$

(iv) $U_{n-m} \in \mathfrak{D}\left\|U_{n-m}\right\|=1$.

(v) $\left(W_{n}, U_{k}\right)$ exists for each $k=1,2, \cdots, n-1$, and $\left(W_{n}, U_{n-m}\right) \neq 0$.

(vi) The solution space $\mathfrak{M}$ of (2.2) in the manifold $\left\{U_{i}\right\}$ is spanned by the $m$ functions $U_{n-m}, U_{n-m+1}, \cdots, U_{n-1}$.

It will be convenient to use the notation

$$
V_{j}=U_{n-m+j} \quad(j=0,1, \cdots, m) .
$$

The assumptions (i), (ii) correspond to those for class 1 problems in [9]. When $n=2$, assumption (iii) is implied by (i), and (v) is implied by (iv). Concerning (iv), it may be that not only $U_{n-m}$, but a subset $\mathfrak{R}$ of $\mathfrak{M}$ with $\operatorname{dim} \mathfrak{R}>1$ lies in $\mathfrak{D}$. However, our attention will be focused on the function $U_{n-m}$, in fact the minimal element of the chain $U_{1} \prec U_{2} \prec \cdots \prec U_{n}$ which lies in D. The function $U_{n-m}$ will be called a basic characteristic function for $T$, corresponding to the characteristic value $A$. It can be shown that under the assumptions, the characteristic values are isolated numbers.

The Wronskian determinant $W$ of the functions $U_{j}(x)$ is a constant because of Abel's formula [4], since $p_{n-1}(x)=0$, and without loss of generality 


$$
W=\operatorname{det}\left(U_{j}^{(i-1)}\right)=-1 \quad(i, j=1,2, \cdots, n) .
$$

The perturbed transformation $A_{\text {s }}$ will now be defined as a restriction of $L$ to functions $y$ satisfying a set of $m$ homogeneous boundary conditions at $x=\varepsilon$, of the form

$$
\begin{aligned}
\mathscr{S}_{\varepsilon i}[y]= & \sum_{j=1}^{n} \alpha_{i j}(\varepsilon) y^{(j-1)}(\varepsilon)=0 \\
& 0<\varepsilon<b \quad(i=1,2, \cdots, m),
\end{aligned}
$$

where the $\alpha_{i j}$ are real-valued functions of $\varepsilon$, and at least one of the functions $\alpha_{i 1}, \alpha_{i 2}, \cdots \alpha_{i n}$ does not vanish for any $\varepsilon$. It will be necessary to assume that these functions satisfy some mild conditions, which will be stated below.

Let $\left|\mathscr{S}_{\varepsilon_{i}}\right|$ denote the boundary operator defined by

$$
\left|\mathscr{B}_{\varepsilon i}\right|[y]=\sum_{j=1}^{n}\left|\alpha_{i j}(\varepsilon)\right|\left|y^{(j-1)}(\varepsilon)\right| \quad(i=1,2, \cdots m) .
$$

Let $|\operatorname{det}|\left(a_{i j}\right)$ denote the sum of the absolute values of the $m$ ! terms in the expansion of the determinant of an order-m matrix $\left(a_{i j}\right)$. Consider the matrix $\left(b_{i k}\right)$ with elements given by

$$
b_{i k}=b_{i k}(\varepsilon)=\mathscr{C}_{\varepsilon i}\left[V_{k}\right] \quad(i, k=1,2, \cdots m),
$$

where the functions $V_{k}$ are given by (2.8). Let $\left(d_{i k}\right)$ be the matrix obtained from $\left(b_{i k}\right)$ by replacing the $h$ th column vector $\left(b_{i k}\right)$ by the vector $\left(-b_{i 0}\right)=\left(-\mathscr{B}_{\varepsilon i}\left[V_{0}\right]\right)$ (the dependence of $\left(d_{i k}\right)$ on $h$ will not be indicated in the notation); and let $\left(e_{i k}\right)$ be obtained from $\left(b_{i k}\right)$ by replacing $\left(b_{i h}\right)$ by any vector whose components are dominated by those of $\left(\left|\mathscr{S}_{\varepsilon i}\right|\left[U_{n}\right]\right), \varepsilon \leq \varepsilon_{0}$.

Assumptions. It will be assumed that the functions

$$
\frac{V_{h} \operatorname{det}\left(d_{i k}\right)}{V_{0} \operatorname{det}\left(b_{i k}\right)} ; \frac{V_{h}|\operatorname{det}|\left(e_{i k}\right)}{V_{m} \operatorname{det}\left(b_{i k}\right)} ; \frac{U_{n} \mathscr{B}_{\varepsilon i}\left[U_{j}\right]}{U_{j} \mathscr{\mathscr { B }}_{\varepsilon}\left[U_{n}\right]}
$$

are bounded functions of $\varepsilon$ whenever $0<\varepsilon \leq \varepsilon_{0}(j=1,2, \cdots n-1 ; h=$ $1,2, \cdots m)$, and in particular

$$
\operatorname{det}\left(b_{i k}\right) \neq 0 \quad 0<\varepsilon \leq \varepsilon_{0} .
$$

Definiton 4. The perturbed domain $\mathfrak{D}_{z}$ is the set of all $u \in \mathfrak{S}$ satisfying the following conditions

(a) $u \in \mathscr{C}^{n-1}(\varepsilon, b)$ and $u^{(n-1)}$ is absolutely continuous on $[\varepsilon, b]$.

(b) $L u \in \mathfrak{S}$

(c) $u$ satisfies the $n-\mathrm{m}$ boundary condition (2.2) at $x=b$.

(d) $u$ satisfies the $m$ boundary conditions (2.10) at $x=\varepsilon$. 
(e) $u$ is identically zero on $(0, \varepsilon)$.

The perturbed operator $A_{\varepsilon}$ is then defined to have domain $\mathcal{D}_{\varepsilon}$ and

$$
A_{\varepsilon} u=L u \quad u \in \mathfrak{D}_{\varepsilon} .
$$

The domain $\mathfrak{D}_{z}$ is to be considered as a perturbation of the basic domain $\mathfrak{D}$, and the perturbation is due to the boundary conditions (d) of Definition 4 being adjoined. In a sense, the operators $A_{\varepsilon}$ converge to the basic operator $T$ as $\varepsilon \rightarrow 0$ [8]. Our problem is to show that the characteristic values and functions of $A_{\varepsilon}$ converge to those of $T$, and furthermore to obtain asymptotic representations which are valid for small values of $\varepsilon$.

3. The comparison procedure. The characteristic value problem under consideration is

$$
L u=\lambda u \quad u \in \mathfrak{D}_{\mathfrak{s}} .
$$

Let $\left\{U_{j}\right\}$ be the fundamental set of solutions of $L y=\Delta y$ which was postulated in $\S 2$. Let $V_{0}=U_{n-m}$ be the basic characteristic function corresponding to the characteristic value $\Lambda$, that is

$$
L V_{0}=\Lambda V_{0} \quad V_{0} \in \mathfrak{D} \quad\left\|V_{0}\right\|=1 .
$$

Let $U$ be any function in the manifold spanned by the $n$ functions $U_{j}$. Let $u=u(x, \lambda)$ be defined by the integral equation

$$
\begin{gathered}
u(x, \lambda)=U(x, \Lambda)+(\Lambda-\lambda) \int_{x}^{b} G(x, t ; \Lambda) u(t, \lambda) d t \\
\text { for } \varepsilon \leq x \leq b, \text { and let } \\
u(x, \lambda)=0 \text { for } 0<x<\varepsilon,
\end{gathered}
$$

where the function

$$
G(x, t ; \Lambda)=\sum_{h=1}^{n} W_{h}(t, \Lambda) U_{h}(x, \Lambda)
$$

is obtained by the classical method of variation-of-parameters [4], and $W_{h}$ is given by (2.5).

Lemma 1. If $U \in \mathscr{C}^{n}(\varepsilon, b)$, then for each fixed value of $\lambda$ and $\varepsilon$ there exists a solution $u$ of (3.3) in $\mathscr{C}^{n}(\varepsilon, b)$. This solution satisfies the differential equation (3.1), and furthermore satisfies the $n-m$ boundary conditions (2.2) at $x=b$ if $U$ satisfies these same conditions.

The proof is well-known [2]. In the sequel $U$ will lie in the solution space of (2.2) in the manifold spanned by $\left\{U_{i}\right\}(i=1,2, \cdots n)$. Then $U$ will have the form 


$$
U(x)=V_{0}(x)+\gamma_{1} V_{1}(x)+\cdots+\gamma_{m-1} V_{m-1}(x)
$$

where the numbers $\gamma_{j}$ are independent of $x$ but depend on $\varepsilon$. The $\gamma_{j}^{\prime} \mathrm{s}$ are to be determined from the boundary conditions (2.10).

Lemma 2. If the numbers $\gamma_{1}, \gamma_{2}, \cdots, \gamma_{m-1}, \lambda$ can be determined so that the solution $u$ of the integral equation (3.3), with $U$ given by (3.5), satisfies the $m$ boundary conditions (2.10), then $\lambda$ is a characteristic value for $A_{2}$ and $u$ is the corresponding characteristic function.

For $u$ satisfies all the conditions of Definition 4 , so that $u \in \mathfrak{D}_{\varepsilon}$, and by Lemma $1, L u=\lambda u$.

Let $H$ be the integral operator defined by

$$
H f(x)=\int_{x}^{b} G(x, t ; \Lambda) f(t) d t
$$

and let $H^{j}$ be the $j$ th iterate of $H$. The solution of (3.3) can then be expressed in the form

$$
u(x)=U(x)+\sum_{j=1}^{\infty}(\Lambda-\lambda)^{j} H^{j} U(x),
$$

which is uniformly convergent and termwise differentiable up to order $n$ (according to Lemma 1). Application of the boundary conditions (2.10) gives the set of $m$ equations

$$
0=\mathscr{S}_{\varepsilon i}[u]=\mathscr{P}_{i i}[U]+\sum_{j=1}^{\infty}(\Lambda-\lambda)^{j} \mathscr{B}_{\varepsilon i}\left[H^{j} U\right]
$$

Define for convenience

$$
\gamma_{m}=\Lambda-\lambda, \quad \gamma_{0}=1 .
$$

Then by (3.5), equations (3.8) can be written in the form

$$
\begin{aligned}
& -\mathscr{P}_{\varepsilon i}\left[V_{0}\right]=\sum_{k=1}^{m-1} \gamma_{k} \mathscr{D}_{\varepsilon i}\left[V_{k}\right]+\gamma_{m} \mathscr{\mathscr { S }}_{\varepsilon i}\left[H V_{0}\right] \\
& \quad+\gamma_{m} \sum_{k=1}^{m-1} \gamma_{k} \mathscr{D}_{\varepsilon i}\left[H V_{k}\right]+\sum_{j=2}^{\infty} \sum_{k=0}^{m-1} \gamma_{k} \gamma_{m}^{j} \mathscr{D}_{\varepsilon i}\left[H^{j} V_{k}\right] \quad(i=1,2, \cdots m),
\end{aligned}
$$

These are power series in $\gamma_{1}, \gamma_{2}, \cdots \gamma_{m}$. Our problem is to invert them, but first certain preliminary results concerning the size of the coefficients in (3.10) will be established. These results will be given in a sequence of lemmas.

LEMma 3. The following asymptotic forms are valid

$$
H U_{k}(x)=\Omega_{k} U_{n}(x)+o\left[U_{n}(x)\right]
$$


as $x \rightarrow 0, k=1,2, \cdots, n-1$, where

$$
\Omega_{k}=\left(W_{n}, U_{k}\right) .
$$

Proof. According to (3.4) and (3.6)

$$
\begin{aligned}
\frac{H U_{k}(x)-\Omega_{k} U_{n}(x)}{U_{n}(x)}= & \sum_{h \neq n} \frac{U_{h}(x)}{U_{n}(x)} \int_{x}^{b} W_{h}(t) U_{k}(t) d t \\
& -\int_{0}^{x} W_{n}(t) U_{k}(t) d t .
\end{aligned}
$$

Since $\left(W_{n}, U_{k}\right)$ exists by assumption (v), the last term on the right side of (3.13) is $o(1)$ as $x \rightarrow 0$. By assumption (ii), each term in the summation is also $o(1)$ as $x \rightarrow 0$.

The following notation will be used:

$$
\begin{aligned}
& \hat{U}(x) \quad=\underset{k}{\operatorname{maximum}}\left\{\left|U_{k}(x)\right|\right\} \\
& \begin{aligned}
\hat{U}^{(h)}(x) & =\underset{k}{\operatorname{maximum}}\left\{\left|U_{k}^{(h)}(x)\right|\right\} \\
\hat{W}(x)=\underset{k}{\operatorname{maximum}}\left\{\left|W_{k}(x)\right|\right\} & \\
0<x \leq b & x_{0} \leq x \leq b \\
g(x) & =\int_{x}^{b} \hat{W}(t) \hat{U}(t) d t \\
& =\int_{x}^{x_{0}}\left|W_{n}(t)\right| \hat{U}(t) d t+g\left(x_{0}\right) \quad 0<x \leq x_{0},
\end{aligned}
\end{aligned}
$$

where $x_{0}$ is a positive number, as postulated in (2.4), (2.7). The function $g(x)$ is uniformly bounded for $x_{0} \leq x \leq b$.

Lemma 4. There is a constant $C$ independent of $x$ and $j$ so that

$$
\begin{aligned}
& \left|H^{j} U_{k}(x)\right| \leq C \frac{[n g(x)]^{j-1}}{(j-1) !} \hat{U}(x) \\
& 0<x \leq b, \quad k=1,2, \cdots, n-1 ; \quad j=1,2 \cdots .
\end{aligned}
$$

Proof. Suppose that $x_{0} \leq x \leq b$. Then it follows easily from (3.4), (3.6), and (3.15) that

$$
\left|H U_{k}(x)\right| \leq n g(x) \hat{U}(x) .
$$

Hence (3.16) is true for $j=1$. Under the assumption that it is true for $j$, it follows that 


$$
\begin{aligned}
\left|H^{j+1} U_{k}(x)\right| & \leq \sum_{i=1}^{n} \int_{x}^{b}\left|W_{i}(t) U_{i}(x) C \frac{[n g(t)]^{j-1}}{(j-1) !} \hat{U}(t)\right| d t \\
& \leq C \frac{n^{j}}{(j-1) !} \hat{U}(x) \int_{x}^{b} \hat{W}(t) \hat{U}(t)[g(t)]^{j-1} d t \\
& =C \frac{n^{j}}{j !}[g(x)]^{j} \hat{U}(x) .
\end{aligned}
$$

Therefore (3.16) is valid by mathematical induction.

Suppose now that $0<x \leq x_{0}$. Then

$$
\begin{aligned}
\left|H U_{k}(x)\right| \leq & \sum_{i=1}^{n} \int_{x}^{x_{0}}\left|W_{i}(t) U_{i}(x) U_{k}(t)\right| d t \\
& +\sum_{i=1}^{n} \int_{x_{0}}^{b}\left|W_{i}(t) U_{i}(x) U_{k}(t)\right| d t \\
\leq & n \underset{k}{\operatorname{maximum}} \int_{x}^{x_{0}}\left|W_{n}(t) U_{n}(x) U_{k}(t)\right| d t+n g\left(x_{0}\right) \hat{U}(x) .
\end{aligned}
$$

It follows from assumption (v), below (2.7), that (3.16) is true for $j=1$. Proceeding by induction.

$$
\begin{aligned}
\left|H^{j+1} U_{k}(x)\right| \leq & \sum_{i=1}^{n} \int_{x}^{x_{0}}\left|W_{i}(t) U_{i}(x) C \frac{[n g(t)]^{j-1}}{(j-1) !} \hat{U}(t)\right| d t \\
& +\sum_{i=1}^{n} \int_{x_{0}}^{b}\left|W_{i}(t) U_{i}(x) C \frac{[n g(t)]^{j-1}}{(j-1) !} \hat{U}(t)\right| d t \\
\leq & C \frac{n^{j}}{(j-1) !} \int_{x}^{x_{0}}\left|W_{n}(t) U_{n}(x)[g(t)]^{j-1} \hat{U}(t)\right| d t \\
& +C \frac{n^{j}}{(j-1) !} \int_{x_{0}}^{b}\left|\hat{W}(t) \hat{U}(x)[g(t)]^{j-1} \hat{U}(t)\right| d t \\
\leq & C \frac{n^{j}}{j !}[g(x)]^{j} \hat{U}(x) .
\end{aligned}
$$

This completes the proof of Lemma 4 .

Lemma 5. For the $m$ boundary operators $\mathscr{D}_{\varepsilon i}[y]$ defined by (2.10), the following asymptotic forms are valid:

$$
\mathscr{\mathscr { S }}_{\varepsilon i}\left[H U_{k}\right]=\Omega_{k} \mathscr{\mathscr { S }}_{\varepsilon i}\left[U_{n}\right]+\mathscr{\mathscr { S }}_{\varepsilon i}\left[U_{n}\right] o(1)
$$

as $\varepsilon \rightarrow 0, i=1,2, \cdots, m ; k=1,2, \cdots, n-1$.

Proof. It is permissible to differentiate $H U_{k}(x)$ under the integral sign. Hence 


$$
\begin{aligned}
\frac{\mathscr{B}_{\varepsilon i}\left[H U_{k}\right]-\Omega_{k} \mathscr{B}_{\varepsilon i}\left[U_{n}\right]}{\mathscr{B}_{\varepsilon}\left[U_{n}\right]}= & -\int_{0}^{\varepsilon} W_{n}(t) U_{k}(t) d t \\
& =\sum_{n \neq n} \frac{\mathscr{B}_{\varepsilon i}\left[U_{h}\right]}{\mathscr{S}_{\varepsilon i}\left[U_{n}\right]} \int_{\varepsilon}^{b} W_{h}(t) U_{k}(t) d t .
\end{aligned}
$$

The first term on the right side is $o(1)$ as in Lemma 3 . Each term in the summation may be decomposed into the factors

$$
\frac{U_{h}(\varepsilon)}{U_{n}(\varepsilon)} \int_{\varepsilon}^{b} W_{h}(t) U_{k}(t) d t, \frac{U_{n}(\varepsilon) \mathscr{B}_{\varepsilon i}\left[U_{h}\right]}{U_{h}(\varepsilon) \mathscr{B}_{\varepsilon i}\left[U_{n}\right]}
$$

The first factor is $o(1)$ as $\varepsilon \rightarrow 0$ by (2.6), and the second factor is bounded by hypothesis (2.12). These considerations establish (3.17).

It will be convenient to introduce, in addition to (2.11), a matrix $\left(c_{i k}\right)$ defined by

$$
\begin{array}{lr}
c_{i k}(\varepsilon)=b_{i k}(\varepsilon) & \text { for } k=1,2, \cdots, m-1 \\
c_{i m}(\varepsilon)=\mathscr{S}_{\varepsilon i}\left[H V_{0}\right] & (i=1,2, \cdots, m) .
\end{array}
$$

Then (3.17) becomes, for $k=n-m$,

$$
\begin{aligned}
& c_{i m}(\varepsilon)=\Omega_{n-m} b_{i m}(\varepsilon)+o\left[b_{i m}(\varepsilon)\right] \\
& \text { as } \varepsilon \rightarrow 0,
\end{aligned}
$$$$
i=1,2, \cdots, m \text {. }
$$

An analogue of Lemma 4 will now be stated. The proof is similar to that of Lemma 4, and will be omitted.

Lemma 6. There is a constant $C$ independent of $x$ and $j$ so that

$$
\left|\left[H^{j} U_{k}(x)\right]^{(h-1)}\right| \leq C \frac{[n g(x)]^{j-1}}{(j-1) !} \hat{U}^{(n-1)}(x)
$$

$$
\begin{gathered}
\left|\mathscr{\mathscr { B }}_{\varepsilon i}\left[H^{j} U_{k}\right]\right| \leq C \frac{[n g(\varepsilon)]^{j-1}}{(j-1) !}\left|\mathscr{\mathscr { B }}_{\varepsilon i}\right|[\hat{U}] \\
0<\varepsilon \leq \varepsilon_{0}(i=1,2, \cdots, m ; k=1,2, \cdots, n-1 ; j=1,2, \cdots)
\end{gathered}
$$

where $\left|\mathscr{B}_{\varepsilon i}\right|$ is defined below (2.10) and $\hat{U}$ is defined by (3.14).

4. Characteristic values and functions. The problem has been reduced to investigating the solutions $\gamma_{j}(j=1,2, \cdots, m)$ of the system (3.10). The quantity $\Lambda-\gamma_{m}$, depending on $\varepsilon$, is the characteristic value $\lambda$ under consideration. The system (3.10) can be written in the form

$$
-b_{i 0}=\sum_{k=1}^{m} c_{i k} \gamma_{k}+\sum C_{i, j_{1} j_{2} \ldots j_{m}} \gamma_{1}^{j} \gamma_{2^{2}}^{j_{2}} \cdots \gamma_{m}^{i_{m}}
$$


where the last summation is extended over indices $j_{1}, j_{2}, \cdots j_{m}$ with the properties

$$
j_{1} \leq 1, j_{2} \leq 1, \cdots, j_{m-1} \leq 1 ; j_{m} \geq 1 \text {. }
$$

The coefficients $c_{i k}$ are given by (3.21), (2.11), and the coefficients $C_{i}, j_{1}, j_{2}, \cdots, j_{m}$ are of the form $\mathscr{D}_{\varepsilon}\left[H^{j_{m}} V_{k}\right]\left(j_{m}=1,2, \cdots ; k=0,1, \cdots, m-1\right)$. The latter will sometimes be abbreviated by $C_{i, \ldots}$.

It follows from Lemma 6 that

$$
\begin{aligned}
& \left|C_{i, \ldots, j_{m}}\right| \leq \frac{[n g(\varepsilon)]_{m}^{j_{m}-1}}{\left(j_{m}-1\right) !}\left|\mathscr{S}_{\varepsilon i}\right|[\hat{U}] \\
& 0<\varepsilon \leq \varepsilon_{0}, \quad i=1,2, \cdots, m ; j_{m}=1,2, \cdots .
\end{aligned}
$$

The determinant of the linear system

$$
-b_{i 0}=\sum_{k=1}^{m} c_{i k} \gamma_{k} \quad(i=1,2, \cdots m)
$$

associated with (4.1) has, on account of (3.18), (3.19) the following asymptotic behaviour

$$
\operatorname{det}\left(c_{i k}\right)=\Omega_{n-m} \operatorname{det}\left(b_{i k}\right)[1+o(1)] \text { as } \varepsilon \rightarrow 0 \quad(i, k=1,2, \cdots, m) .
$$

Since $\Omega_{n-m} \neq 0$ by hypothesis (v), and since $\operatorname{det}\left(b_{i k}\right) \neq 0$ by (2.13), the linear system (4.4) possesses unique solutions $\gamma_{k}$ for $0<\varepsilon \leq \varepsilon_{0}$. Hence (4.1) can be written in the form

$$
\gamma_{h}=\nu_{h}+\sum D_{h}, \cdots, \gamma_{1}^{j} \gamma_{2}^{j} \cdots \gamma_{m}^{j} \quad(h=1,2, \cdots, m)
$$

where

$$
\nu_{h}=\frac{\operatorname{det}\left(d_{i k}\right)}{\Omega_{n-m} \operatorname{det}\left(b_{i k}\right)}[1+o(1)] \text { as } \varepsilon \rightarrow 0
$$

and

$$
D_{h}, \cdots=\frac{\operatorname{det}\left(a_{i k}\right)}{\Omega_{n-m} \operatorname{det}\left(b_{i k}\right)}[1+o(1)] \text { as } \varepsilon \rightarrow 0
$$

Here, the matrices $\left(b_{i k}\right),\left(d_{i k}\right)$ have been defined in (2.11)-(2.12), and $\left(a_{i k}\right)$ is defined as follows

$$
\begin{aligned}
a_{i k} & =b_{i k} \quad \text { for } k=1,2, \cdots, m, k \neq h \\
& =C_{i}, \cdots \text { for } k=h .
\end{aligned}
$$

The result (4.5) has been used in obtaining the asymptotic forms (4.7), (4.8). 
It follows from the assumption (2.12), and from (4.3), (4.7), and (4.8) that there exists a constant $C$ so that

$$
\begin{gathered}
\left|\nu_{h}(\varepsilon)\right| \leq C\left|V_{0}(\varepsilon) / V_{h}(\varepsilon)\right| \\
\left|D_{h, \cdots j_{m}}\right| \leq C \frac{[C g(\varepsilon)]_{m}^{j^{-1}}}{\left(j_{m}-1\right) !} \frac{|\operatorname{det}|\left(e_{i k}\right)}{\left|\Omega_{n-m} \operatorname{det}\left(b_{i k}\right)\right|} \\
\leq \frac{[C g(\varepsilon)]^{j} m^{-1}\left|V_{m}(\varepsilon)\right|}{\left(j_{m}-1\right) !\left|V_{h}(\varepsilon)\right|} \\
0<\varepsilon \leq \varepsilon_{0}, h=1,2, \cdots, m ; j_{m}=1,2, \cdots .
\end{gathered}
$$

Let new variables $s_{h}$ be introduced by the relations

$$
\gamma_{h}=\nu_{h} s_{h} \quad(h=1,2, \cdots, m) .
$$

Then the system (4.6) becomes

$$
s_{h}=1+\sum E_{h}, \cdots, s_{1}^{j} s_{2^{2}}^{j} \cdots s_{m^{m}}^{j}
$$

where

$$
E_{h}, \cdots=D_{h}, \cdots, \nu_{1}^{j} \nu_{2}^{j} \cdots \nu_{h}^{j} h^{-1} \cdots \nu_{m}^{j} m .
$$

Lemma 7. The system (4.12) possesses unique solutions $s_{h}(\varepsilon)$ in the neighborhood of $\varepsilon=0$, which may be represented by the convergent series

$$
s_{h}=1+\sum_{k=2}^{\infty} B_{h k},
$$

where the coefficients $B_{h k}$ are obtained by formal substitution of the $m$ power series

$$
s_{h}=z+\sum_{k=2}^{\infty} B_{h k} z^{k}
$$

into the system

$$
s_{h}=z+\sum E_{h}, \cdots, s_{1}^{j} s_{2}^{j} \cdots s_{m}^{j} m
$$

and equating the coefficients of each power of $z$.

Proof. The Cauchy majorization procedure [3, p. 470] will be used to show that (4.16) has solutions $s_{h}$ representable by power series expansions, convergent when $z=1$. Accordingly, (4.16) can be dominated by the system

$$
S_{h}=S=Z-\left[1+R^{-1}\left(S_{1}+\cdots+S_{m}\right)\right]+\left[1-R^{-1}\left(S_{1}+\cdots S_{m}\right)\right]^{-1}
$$


provided $R$ is chosen suitably. In the power series expansion of the right side of (4.17), the sum of the exponents for any term is $\geq 2$, as is the case also in (4.16).

It can be seen from (4.9), 4.10), and (4.13) that the system (4.16) is dominated by (4.17) whenever $R^{-1}$ is of order $\nu_{1}(\varepsilon) g(\varepsilon)$, which is $o(1)$ as $\varepsilon \rightarrow 0$ by (2.6), (3.15). Then, according to the Cauchy majorization procedure, the series (4.15) for the solutions of (4.16) are dominated by the series for the solutions $S_{h}$ of (4.17). However, the latter are easily constructed, as follows:

$$
S_{h}=\frac{R(R+m Z)}{2\left(R m+m^{2}\right)}\left[1-\left(1-\frac{4\left(R m+m^{2}\right) Z}{(R+m Z)^{2}}\right)^{1 / 2}\right],
$$

and the right member is developable in a power series about $Z=0$, with radius of convergence $Z_{0}$ of order $\left(\nu_{1} g\right)^{-1}$. Then the $m$ series (4.14), obtained from (4.15) when $z=1$, are convergent series expansions for the solutions of the system (4.12).

LEMma 8. The series on the right side of (4.14) constitutes an asymptotic expansion of $s_{h}(\varepsilon)$ with scale $\delta=\nu_{1}(\varepsilon) g(\varepsilon)$, as $\varepsilon \rightarrow 0$. In particular, the following asymptotic forms are valid:

$$
s_{h}(\varepsilon)=1+o(1) \text { as } \varepsilon \rightarrow 0 \quad(h=1,2, \cdots, m) .
$$

Proof. The coefficients $B_{l l k}$ in (4.14) are dominated by the coefficients in the power series expansion of (4.18). Hence

$$
\sum_{k=2}^{\infty}\left|B_{h k}\right|=O\left(R^{-1}\right)=O\left[\nu_{1}(\varepsilon) g(\varepsilon)\right]
$$

which establishes (4.19).

The coefficients $B_{h k}$, as determined by formal substitution of (4.15) into (4.16), have the form

$$
B_{h k}=\sum \Phi E_{h, j_{1}, j_{2}, \cdots, i} B_{1 q_{1}} B_{2 q_{2}} \cdots\left(B_{m m_{1}} B_{m m_{2}} \cdots B_{m m_{i}}\right)
$$

where the summation extends over indices

$$
j_{1}, j_{2}, \cdots, i ; q_{1}, q_{2}, \cdots, q_{m-1} ; m_{1}, m_{2}, \cdots, m_{i}
$$

with the properties

$$
\begin{aligned}
& j_{1} \leq 1, j_{2} \leq 1, \cdots, i=1,2, \cdots ; \\
& q_{1}+q_{2}+\cdots+q_{m-1}+m_{1}+m_{2}+\cdots+m_{i}=k .
\end{aligned}
$$

The coefficients $\Phi$ depend on the indices but not on $\varepsilon$. It is understood that $B_{h 1}=1$. It follows from (4.13) that 


$$
E_{h, j_{1}, j_{2}, \ldots, i}=O\left(\nu_{1^{1}+j_{2}+\ldots+i-1}\right) g^{i-1} .
$$

Then an inductive argument shows that $B_{l k}=O\left(\delta^{k-1}\right)(h=1,2, \cdots, m ; k=$ $1,2, \cdots)$. Hence by (4.14),

$$
s_{h}-1-B_{h 2}-\cdots-B_{h k}=O\left(\delta^{k}\right),
$$

so that the series on the right side of (4.14) is an asymptotic expansion for $s_{h}$ with scale $\delta(\varepsilon)$ as $\varepsilon \rightarrow 0$.

According to (4.11), (4.14), the solutions of (4.6) are

$$
\gamma_{h}(\varepsilon)=\nu_{h}(\varepsilon)\left[1+\sum_{k=2}^{\infty} B_{h k}(\varepsilon)\right] \quad(h=1,2, \cdots, m)
$$

and the right member constitutes an asymptotic expansion for $\gamma_{h}(\varepsilon)$ as $\varepsilon \rightarrow 0$ for each $h=1,2, \cdots, m$. Evidently (4.21) are also solutions of (4.1) and hence of (3.10). In particular the following asymptotic forms are valid:

$$
\gamma_{h}(\varepsilon)=\nu_{h}(\varepsilon)[1+o(1)] \text { as } \varepsilon \rightarrow 0
$$

THEOREM 1. For each characteristic value $\Lambda$ of the basic operator $T$, there exists a characteristic value $\lambda(\varepsilon)$ of the perturbed operator $A_{\varepsilon}$ which converges to $\Lambda$ as $\varepsilon \rightarrow 0$, and furthermore $\lambda(\varepsilon)$ has the convergent asymptotic expansion

$$
\lambda(\varepsilon)=\Lambda-\nu_{m}(\varepsilon)\left[1+\sum_{k=2}^{\infty} B_{m k}(\varepsilon)\right]
$$

with scale $\nu_{1} g$ as $\varepsilon \rightarrow 0$

Proof. The coefficients $\gamma_{h}$ given by (4.21) have been determined so that the function $u$, with the representation (3.7), satisfies (3.10), or (2.10). Then Lemma 2 shows that $\lambda(\varepsilon)=\Lambda-\gamma_{m}(\varepsilon)$ is a characteristic value for $A_{\varepsilon}$, and that $u$ is the corresponding characteristic function. The expansion (4.23) follows from (4.21).

In particular, the following asymptotic form is valid

$$
\lambda(\varepsilon)=\Lambda-\nu_{m}(\varepsilon)[1+o(1)] \text { as } \varepsilon \rightarrow 0 .
$$

An asymptotic expansion for the characteristic function $u=u(x, \lambda)$ corresponding to $\lambda$ can now be obtained directly from (3.7); for it follows from (3.7), (3.16), (4.23) that

$$
\begin{aligned}
& u(x, \lambda)-U(x, \Lambda)-\sum_{j=1}^{N}\left[\gamma_{m}(\varepsilon)\right]^{\jmath} H^{j} U(x) \\
& =\gamma_{m}(\varepsilon) \hat{U}(x, \Lambda) O\left\{\left[\gamma_{m}(\varepsilon) g(x)\right]^{N}\right\} \\
& \varepsilon \leq \varepsilon_{0}, \varepsilon \leq x \leq b, N=1,2, \cdots .
\end{aligned}
$$


Furthermore, $\gamma_{m}(\varepsilon) g(x)=o(1)$ as $\varepsilon \rightarrow 0$, uniformly for $\varepsilon \leq x \leq b$, on account of (2.6), (3.15), (4.9), and (4.22). Then $u$ has the asymptotic expansion

$$
u(x, \lambda)-U(x, \Lambda) \sim \sum_{j=1}^{\infty}\left[\gamma_{m}(\varepsilon)\right]^{j} H^{j} U(x)
$$

with scale $\delta=\gamma_{m}(\varepsilon) g(x)$ as $\varepsilon \rightarrow 0$, valid uniformly for $\varepsilon \leq x \leq b$. The function $\alpha$ in Definition 2 can be taken to be $\gamma_{m}(\varepsilon) \hat{U}(x, \Lambda)$. The function $U(x, \Lambda)$, depending on $\varepsilon$, is defined by (3.7).

In particular, the result

$$
u(x, \lambda)=V_{0}(x, \Lambda)+\sum_{k=1}^{m-1} \gamma_{k}(\varepsilon) V_{k}(x, \Lambda)+O\left[\gamma_{m}(\varepsilon) \hat{U}(x, \Lambda)\right]
$$

displays the error term $u(x, \lambda)-V_{0}(x, \Lambda)$. When $x$ is in a closed subset $I$ of $\varepsilon \leq x \leq b$ which is independent of $\varepsilon$, the error term is of order $\nu_{1}(\varepsilon)$, or $V_{0}(\varepsilon) / V_{1}(\varepsilon)$, uniformly for $x \in I$. On the other hand, when the ratio $x / \varepsilon$ remains bounded as $\varepsilon \rightarrow 0$, the error term is of order $\gamma_{m}(\varepsilon) U_{n}(x)$, or $V_{0}(\varepsilon)$.

THEOREM 2. The characteristic function $u(x, \lambda)$ corresponding to the characteristic value $\lambda(\varepsilon)$ of the perturbed operator $A_{\varepsilon}$ possesses the uniform asymptotic expansion (4.24), valid as $\varepsilon \rightarrow 0$ over the perturbed interval $\varepsilon \leq x \leq b$.

Some special cases will now be stated. Let $J\left[V_{0}\right]$ denote a closed subset of the interval $(0, b]$ with the property that $V_{0}(x, \Lambda)$ does not vanish whenever $x \in J\left[V_{0}\right]$. Then the asymptotic form

$$
u(x, \lambda)=V_{0}(x, \Lambda)[1+o(1)]
$$

is valid as $\varepsilon \rightarrow 0$, uniformly for $x \in J\left[V_{0}\right]$.

Further, let $J\left[V_{0}, V_{1}, \cdots, V_{k}\right](k=0,1, \cdots, m-1)$ denote a closed subset of $(0, b]$ on which none of the functions $V_{0}, V_{1}, \cdots, V_{k}$ vanish. Then the asymptotic series

$$
u(x, \lambda)=V_{0}(x, \Lambda)+\gamma_{1}(\varepsilon) V_{1}(x, \Lambda)+\cdots+\gamma_{k}(\varepsilon) V_{k}(x, \Lambda)[1+o(1)]
$$

is valid as $\varepsilon \rightarrow 0$, uniformly for $x \in J\left[V_{0}, V_{1}, \cdots, V_{k}\right](k=0,1, \cdots, m-1)$.

5. Regular singularities. In this section, the point $x=0$ is supposed to be a regular singular point for the differential operator (1.1). Specifically, it is assumed that the functions $p_{j}(x)$ have the asymptotic behavior

$$
p_{j}(x) \sim p_{j, j-n} x^{j-n}+p_{j, j-n+1} x^{j-n+1}+\cdots
$$

as $x \rightarrow 0(j=0,1, \cdots, n-2)$, and that $(2.1)$ holds. Let $\pi,(j=1,2, \cdots, n)$ 
denote the $n$ zeros of the polynomial

$$
\sum_{j=1}^{n} p_{j, j-n} \pi(\pi-1) \cdots(\pi-j+1)+p_{0,-n},
$$

where $p_{n, 0}=-1, p_{n-1,-1}=0$, all supposed to be real and distinct, ordered as follows:

$$
\pi_{1}>\pi_{2}>\cdots \pi_{n}
$$

Evidently

$$
\sum_{j=1}^{n} \pi_{j}=1+2+\cdots(n-1) .
$$

Then the differential equation $L y=\Lambda y$ possesses a fundamental set of solutions $U_{j}(x)$ with the asymptotic behavior

$$
U_{j}(x) \sim a_{j} x^{\pi_{j}} \quad x \rightarrow 0 \quad(j=1,2, \cdots, n),
$$

where the constants $a$, are independent of $\Lambda$ as well as $x$.

It follows from (5.2) that the solutions (5.4) are asymptotically ordered as $x \rightarrow 0$, and hence assumption (i) of section (2) is satisfied. Also, according to (2.5) and (5.4),

$$
W_{j}(x) \sim b_{j} x^{\beta} j, \beta_{j}=\sum_{i \neq j} \pi_{i}-1-2-\cdots-(n-2),
$$

and from this it is seen that assumption (ii) is valid. From the asymptotic behavior of $U_{j}$ and $W_{j}$ as $x \rightarrow 0$, it follows that

$$
\frac{\left|U_{i}(x) W_{i}(t)\right|}{\left|U_{n}(x) W_{n}(t)\right|} \leq C\left(\frac{x}{t}\right)^{\pi_{i}-\pi_{n}}
$$

and hence assumption (iii) is also valid. Then in the case that the exponents at the singularity are real and distinct, ordered by (5.2), the assumptions (i), (ii), (iii), are all satisfied independent of the number $\Lambda$. The distinctness assumption could be removed by introducing solutions like $x^{\pi}, x^{\pi} \log x$, corresponding to a double zero $\pi$ [4]. This has been done in [9] for the case $n=2$, but the discussion will be omitted here.

Concerning the boundary conditions (2.10), suppose that each function $\alpha_{i n}(\varepsilon) \neq 0\left(0<\varepsilon \leq \varepsilon_{0} ; i=1,2, \cdots, m\right)$ and that the limits

$$
\sigma_{i \jmath}=\lim _{\varepsilon \rightarrow 0} \frac{\varepsilon^{n-j} \alpha_{i j}(\varepsilon)}{\alpha_{i n}(\varepsilon)}
$$

all exist. Then sufficient conditions for (2.12), (2.13) to hold are

$$
\mu_{i, n} \neq 0 ; \operatorname{det}\left(\mu_{i k}\right) \neq 0
$$

where 


$$
\mu_{i k}=\sigma_{i 1}+\sum_{j=2}^{n} \sigma_{i j} \pi_{k}\left(\pi_{k}-1\right) \cdots\left(\pi_{k}-j+2\right) .
$$

Similar conditions can be obtained when some of the functions $\alpha_{i n}(\varepsilon)$ are identically zero.

Explicit asymptotic forms for $\nu_{h}$ in (4.7) can now be obtained under the assumptions (5.5), (5.6), with the aid of (2.10), (5.4). The results turn out to be

$$
\nu_{h}(\varepsilon)=\omega_{h} \varepsilon^{q} h[1+o(1)], \varepsilon \rightarrow 0(h=1,2, \cdots, m),
$$

where

$$
\omega_{h}=\frac{a_{0}}{a_{h} \Omega_{n-m}} \frac{\operatorname{det}\left(\mu_{i j}\right)}{\operatorname{det}\left(\mu_{i k}\right)} ; q_{h}=\pi_{n-m}-\pi_{n-m+h} .
$$

Here, the index $i$ assumes the values $1,2, \cdots, m ; k$ assumes the values $n-m+1, n-m+2, \cdots, n$; and $j$ assumes the same values as $k$ except $n-m+h$ is replaced by $n-m$. Then asymptotic forms of the type (4.23) can be obtained for the characteristic values $\lambda(\varepsilon)$ as $\varepsilon \rightarrow 0$.

As an example of (2.10), consider the boundary conditions $y^{(i-1)}(\varepsilon)=$ $0(i=1,2, \cdots, m)$. In this case, the matrix $\left(\mu_{i k}\right)$ involved in (5.7) is given by

$$
\begin{aligned}
& \mu_{1 k}=1 \\
& \mu_{i k}=\pi_{k}\left(\pi_{k}-1\right) \cdots\left(\pi_{k}-i+2\right) \quad(i=2,3, \cdots, m),
\end{aligned}
$$

and it can be seen that $\left(\mu_{i k}\right)$ is equivalent to the Van der Monde matrix $\left(\pi_{k}^{i-1}\right)$. Since [6] $\operatorname{det}\left(\pi_{k}^{i-1}\right)=\Pi\left(\pi_{i}-\pi_{k}\right)(i>k)$ it follows that

$$
\frac{\operatorname{det}\left(\mu_{i j}\right)}{\operatorname{det}\left(\mu_{i k}\right)}=\prod_{j} \frac{\pi_{n-m}-\pi_{j}}{\pi_{n-m+h}-\pi_{j}}
$$

where the product is taken over all the values $j=n-m+1, n-m+2$, $\cdots n$, except $n-m+h$. Since $\pi_{j} \neq \pi_{n-m+h}$ for $j \neq n-m+h$ by the distinctness hypothesis, it follows that the asymptotic forms (5.7) for $\nu_{h}(\varepsilon)(h=1,2, \cdots, m)$ can be determined explicitly, and in particular when $h=m$,

$$
\lambda(\varepsilon)=\Lambda-\omega_{m} \varepsilon^{q}[1+o(1)] \text { as } \varepsilon \rightarrow 0 .
$$

For characteristic functions $u=u(x, \lambda)$, a result more precise than (4.24) will be obtained when $x$ is on a closed subset of $(0, b]$. Consider the decomposition

$$
i=j m+k(k=1,2, \cdots, m ; j=0,1,2, \cdots)
$$

of the positive integer $i$. Define 


$$
F_{i}(x, \varepsilon)=\gamma_{m}^{j} \gamma_{k-1} H^{j} U_{k-1}(x, \Lambda)
$$

and

$$
\sigma_{i}=j\left(\pi_{n-m}-\pi_{n}\right)+\left(\pi_{n-m}-\pi_{n-m+k-1}\right) .
$$

Then, according to (3.16) and (5.8),

$$
\left|F_{i}(x, \varepsilon)\right| \leq \frac{\left[n \gamma_{m}(\varepsilon) g(x)\right]^{j}}{(j-1) !} \gamma_{k-1}(\varepsilon) \hat{U}(x)
$$

It follows that whenever $x$ is on a closed subset of $(0, b]$,

$$
\begin{aligned}
& u(x, \lambda)-\sum_{h=1}^{i-1} F_{h}(x, \varepsilon)=O\left\{\left[\gamma_{m}(\varepsilon)\right]^{j} \gamma_{k-1}(\varepsilon)\right\} \\
& =O\left(\varepsilon^{\sigma_{i}}\right) \\
& 0<\varepsilon \leq \varepsilon_{0} \quad(i=1,2, \cdots) \text {. }
\end{aligned}
$$

Then the series $\sum F_{i}(x, \varepsilon)$ represents a uniform asymptotic expansion for the characteristic function $u(x, \lambda)$ as $\varepsilon \rightarrow 0$.

\section{REFERENCES}

1. E. A. Coddington, The spectral representation of ordinary self-adjoint differential operators, Annals of Math. 60 (1954), 192-211.

2. , and N. Levinson, Theory of ordinary differential equations, McGraw-Hill (1955).

3. E. Goursat, Cours d'analyse mathematique I, Gauthier-Villars (1943).

4. E. L. Ince, Ordinary differential equations, Dover (1956).

5. T. Kato, Quadratic forms in Hilbert spaces, and asymptotic perturbation series, Technical report no. 7, Department of Mathematics, University of California, Berkeley (1955).

6. L. Mirsky, An introduction to linear algebra, Oxford (1955).

7. F. Rellich, Spectral theory of a second order ordinary differential operator, New York University (1953).

8. F. Riesz and B. Sz.-Nagy, Functional analysis, Blackie and Son (1956).

9. C. A. Swanson, Differential operators with perturbed domains, J. of rational mechanics and analysis, 6, 6 (1957), 823-846

10. J. G. Van Der Corput, Asymptotic expansions, I. Technical report 1, Department of Mathematics, University of California, Berkeley (1954).

11. H. Weyl, Ueber gewöhnliche Differentialgleichungen mit Singularitäten und die zugehörigen Entwicklungen willkürlichen Funktionen, Math. Ann. 68 (1910), 220-269.

The UNIVERsity of British COLUmbia 


\section{PACIFIC JOURNAL OF MATHEMATICS}

\section{EDITORS}

\section{David Gilbarg}

Stanford University

Stanford, California

\section{R. A. Beaumont}

University of Washington

Seattle 5 , Washington

\author{
A. L. Whiteman
}

University of Southern California Los Angeles 7, California

L. J. Paige

University of California

Los Angeles 24, California

\author{
E. F. BECKENBACH \\ C. E. BURGESS \\ E. HEWITT \\ A. HORN
}

\author{
V. GANAPATHY IYER \\ R. D. JAMES \\ M. S. KNEBELMAN \\ L. NACHBIN
}

ASSOCIATE EDITORS
I. NIVEN

T. G. OSTROM

H. L. ROYDEN

M. M. SCHIFFER
E. G. STRAUS

G. SZEKERES

F. WOLF

K. YOSIDA

\section{SUPPORTING INSTITUTIONS}

\author{
UNIVERSITY OF BRITISH COLUMBIA \\ CALIFORNIA INSTITUTE OF TECHNOLOGY \\ UNIVERSITY OF CALIFORNIA \\ MONTANA STATE UNIVERSITY \\ UNIVERSITY OF NEVADA \\ OREGON STATE COLLEGE \\ UNIVERSITY OF OREGON \\ OSAKA UNIVERSITY \\ UNIVERSITY OF SOUTHERN CALIFORNIA
}

\author{
STANFORD UNIVERSITY \\ UNIVERSITY OF TOKYO \\ UNIVERSITY OF UTAH \\ WASHINGTON STATE COLLEGE \\ UNIVERSITY OF WASHINGTON \\ * * * \\ AMERICAN MATHEMATICAL SOCIETY \\ CALIFORNIA RESEARCH CORPORATION \\ HUGHES AIRCRAFT COMPANY \\ SPACE TECHNOLOGY LABORATORIES
}

Mathematical papers intended for publication in the Pacific Journal of Mathematics should be typewritten (double spaced), and the author should keep a complete copy. Manuscripts may be sent to any one of the four editors. All other communications to the editors should be addressed to the managing editor, L. J. Paige at the University of California, Los Angeles 24, California.

50 reprints per author of each article are furnished free of charge; additional copies may be obtained at cost in multiples of 50 .

The Pacific Journal of Mathematics is published quarterly, in March, June, September, and December. The price per volume (4 numbers) is $\$ 12.00$; single issues, $\$ 3.50$. Back numbers are available. Special price to individual faculty members of supporting institutions and to individual members of the American Mathematical Society: $\$ 4.00$ per volume; single issues, $\$ 1.25$.

Subscriptions, orders for back numbers, and changes of address should be sent to Pacific Journal of Mathematics, 2120 Oxford Street, Berkeley 4, California.

Printed at Kokusai Bunken Insatsusha (International Academic Printing Co., Ltd.), No. 6, 2-chome, Fujimi-cho, Chiyoda-ku, Tokyo, Japan.

PUBLISHED BY PACIFIC JOURNAL OF MATHEMATICS, A NON-PROFIT CORPORATION

The Supporting Institutions listed above contribute to the cost of publication of this Journal, but they are not owners or publishers and have no responsibility for its content or policies. 


\section{Pacific Journal of Mathematics}

\section{Vol. 9, No. $2 \quad$ June, 1959}

Lee William Anderson, On the breadth and co-dimension of a topological lattice

Frank W. Anderson and Robert L. Blair, Characterizations of certain lattices

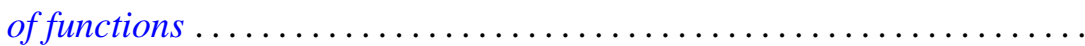

Donald Charles Benson, Extensions of a theorem of Loewner on integral

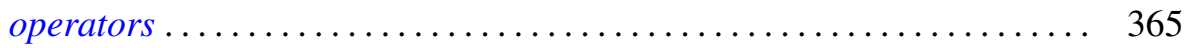

Errett Albert Bishop, A duality theorem for an arbitrary operator ........ 379

Robert McCallum Blumenthal and Ronald Kay Getoor, The asymptotic distribution of the eigenvalues for a class of Markov operators ........

Delmar L. Boyer and Elbert A. Walker, Almost locally pure Abelian

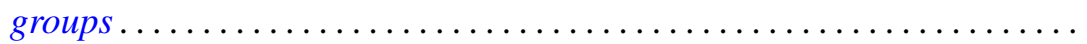

Paul Civin and Bertram Yood, Involutions on Banach algebras ........... Lincoln Kearney Durst, Exceptional real Lehmer sequences .... 415

Eldon Dyer and Allen Lowell Shields, Connectivity of topological

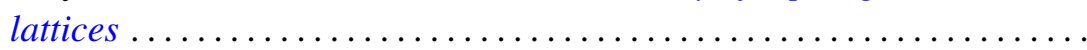

Ronald Kay Getoor, Markov operators and their associated

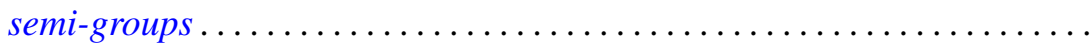

Bernard Greenspan, A bound for the orders of the components of a system of algebraic difference equations

Branko Grünbaum, On some covering and intersection properties in

Minkowski spaces ............................

Bruno Harris, Derivations of Jordan algebras ..............

Henry Berge Helson, Conjugate series in several variables.

Isidore Isaac Hirschman, Jr., A maximal problem in harmonic analysis.

II .

Alfred Horn and Robert Steinberg, Eigenvalues of the unitary part of a matrix

Edith Hirsch Luchins, On strictly semi-simple Banach algebras ...

William D. Munro, Some iterative methods for determining zeros of

functions of a complex variable...

John Rainwater, Spaces whose finest uniformity is metric .

William T. Reid, Variational aspects of generalized convex functions ....

A. Sade, Isomorphisme d'hypergroupoï des isotopes ...... . .

Isadore Manual Singer, The geometric interpretation of a special

connection . . .

Charles Andrew Swanson, Asymptotic perturbation series for characteristic

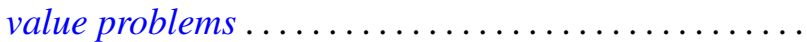

